\title{
Disturbances in the voluntary recruitment order of anterior tibial motor units in spastic paraparesis upon fatigue ${ }^{1}$
}

\author{
LENNART GRIMBY, JAN HANNERZ, AND TYRA RÅNLUND \\ From the Department of Neurology, Karolinska Sjukhuset, Stockholm
}

SUMMARY The recruitment order of motor units in the tibialis anterior muscle upon fatigue of tonic voluntary contraction was studied in 20 patients with severe spastic paraparesis. An electromyographic technique for secure identification of single motor units was used. Before fatigue the recruitment order is stable and low-frequency units are recruited before high-frequency units; this recruitment pattern agrees with that in normal voluntary activity. When fatigue appears, however, the recruitment order becomes indefinite and high-frequency units can be recruited before lowfrequency units; this recruitment pattern agrees with that in normal phasic voluntary activity. Finally, all voluntary activation power disappears, even in the case of units which have never been active. Contraction ability and original recruitment order are restored upon rest but also upon tonic reflex support of the voluntary drive. Whether the fatigue reaction may be due to insufficient gamma motoneurone innervation and whether it is related to spasticity are discussed. The practical physiotherapeutic implications are reviewed.

In animals, certain motor units of skeletal muscle have muscle fibres with long contraction time and high resistance to fatigue, while other motor units have muscle fibres with short contraction time and lower resistance to fatigue. Human motor units should be differentiated in the same way. The recruitment order of human motor units can be determined in electromyographic recordings by the characteristic shape of their potentials. A hint of the contraction time can also be obtained electromyographically, as the discharge frequency of a motor unit should be correlated with its fusion frequency.

We have previously shown that normal human subjects can choose the recruitment order that seems adequate for the work intended. For tonic work, low-frequency units, with presumably long contraction time and high resistance to fatigue, are selected and recruited one by one in a stable order. For phasic work, on the other hand, high-frequency units are activated in a higher degree, but there is a tendency for

1 This investigation was supported by grants from the Swedish Multiple Sclerosis Society, Stockholm. several units to be recruited at the same time and the recruitment order is unstable. For references and detailed background data, see Grimby and Hannerz (1973b).

A series of investigations on voluntary contraction in patients with different neurological diseases is in progress with the aim of determining the patient's ability to select the proper recruitment order for the work intended. In a previous paper the starting difficulties of patients with severe bradykinesia of Parkinsonism were studied (Grimby and Hannerz, 1973b). It was shown that the patients had a decreased ability to change from 'phasic' to 'tonic' recruitment order and vice versa.

In this paper the decreased endurance of flexor muscles in spastic paraplegia is studied. It is shown that the normal 'tonic' recruitment order in sustained voluntary activity is replaced by 'phasic' recruitment order when fatigue appears. It is suggested that the paraparetic patient loses his ability to use the path for voluntary facilitation of the motoneurone pool that is normally used for tonic voluntary activity, and has to de- 
pend on a path that normally is used only for phasic voluntary activity. The role of spasticity and of decreased fusimotor function for the decreased endurance of activity with 'tonic' recruitment order is discussed. A preliminary report was presented at the International EMG Congress in Brussels in September 1971 (Grimby and Hannerz, 1973a).

\section{METHODS}

Twenty patients with severe spastic parapareses were studied. The tibialis anterior muscle was tested. The tonic voluntary maximum power was reduced to $5-25 \%$ of normal maximum power.

The electromyographic recording techniques were the same as those used in previous studies (Grimby and Hannerz, 1973b). The amplifier was connected to a loudspeaker. The patients were instructed to activate the anterior tibial muscle in such a way that a continuous sound was emitted by the loudspeaker and to moderate the contraction so that the sound became as weak as possible. Such a threshold contraction does not interfere with the circulation in the muscle, which has been considered to be a factor that limits the endurance of a contraction in a normal man (Merton, 1954).

\section{RESULTS}

ENDURANCE OF SUSTAINED VOLUNTARY ACTIVITY IN NORMAL SUBJECTS Each motor unit discharges within a particular frequency range. In the anterior tibial muscle this range is $7-25 / \mathrm{sec}$ for a low-frequency unit and 25-65/sec for a high-frequency unit and anything in between for intermediate forms (Hannerz, 1973).

The units first recruited in tonic voluntary activity in normal subjects are of low-frequency type. The recruitment order of motor units in normal tonic voluntary activity is predominantly stable and, when 'rotation' occurs, it is only motor units with identical discharge frequency ranges and nearby thresholds that are involved. The units are called B1, B2, B3, etc., in the order in which they are recruited upon increasing tonic voluntary contraction; this is done in order to conform with previous papers in which the unit first recruited in phasic activity was called the A unit and the unit first recruited in tonic activity the B unit.

A normal subject can always drive the B1 unit voluntarily for hours, even though he sometimes has to concentrate considerably. With the
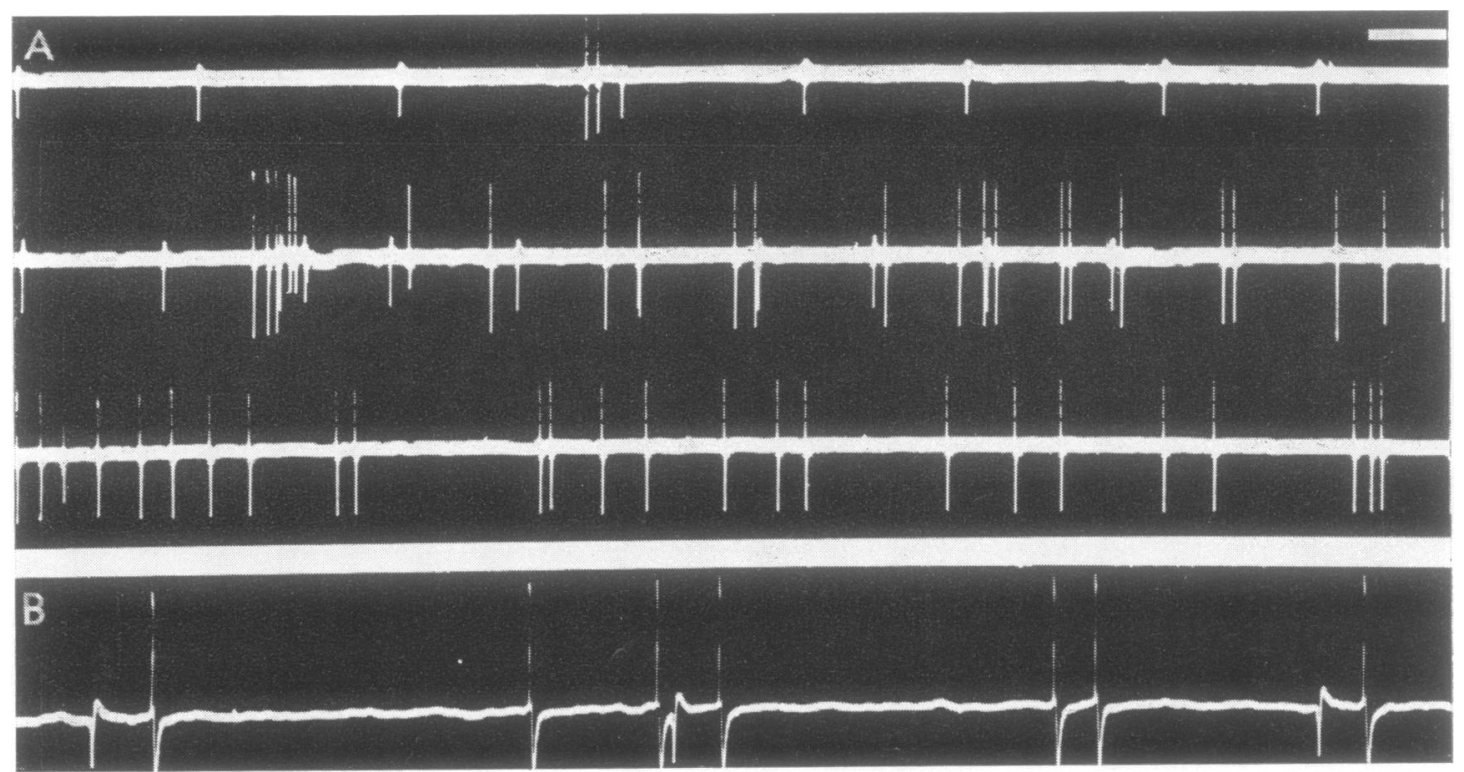

FIG. 1. Change from low-to high-frequency unit upon fatigue. A. Each trace is a direct continuation of the trace above. Time bar $100 \mathrm{msec}$. B. Part of the recording in A at five times faster speed to show the characteristic and constant shape of the two motor unit potentials. 


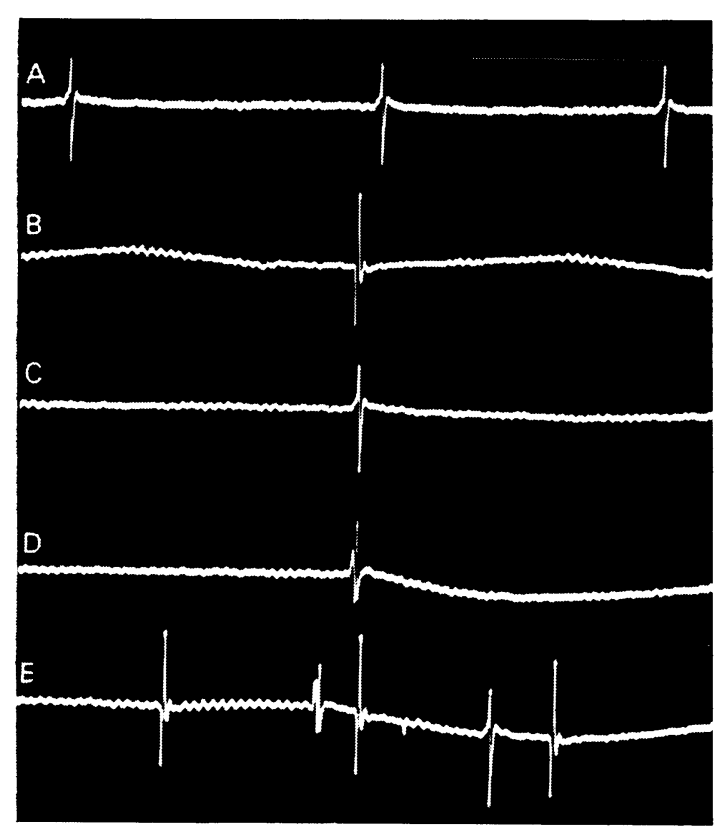

FIG. 2. Change from stable to unstable recruitment order upon fatigue. A. Before fatigue. B-E. Upon fatigue. Time bar $100 \mathrm{msec}$. electrode used, the B1 unit is always active when only a small percentage of maximum muscular power is used. Electromyographic signs of fatigue do not appear in contractions below the $25 \%$ level. This is in agreement with previous investigations.

ENDURANCE OF SUSTAINED VOLUNTARY ACTIVITY IN PARAPARETIC PATIENTS At the beginning of sustained voluntary activity in paretic subjects the results agree with those in normal subjects; the first recruited units B1, B2, B3 discharge within a frequency range of $7-25 / \mathrm{sec}$; the recruitment order is predominantly stable; the B1 unit discharges when only a small percentage of normal muscular power is used.

Severely paraparetic patients, however. are usually able to keep the B1 unit in continuous activity voluntarily for a limited period only. The B1 unit may be lost at any time between 30 seconds and 30 minutes, depending on the following factors:

The more the patient has to concentrate to $\stackrel{0}{\mathbb{D}}$ 글 activate the Bl unit the more rapidly it is fatigued. Conversely, when the B1 unit tends to be active spontaneously no fatigue reaction appears.

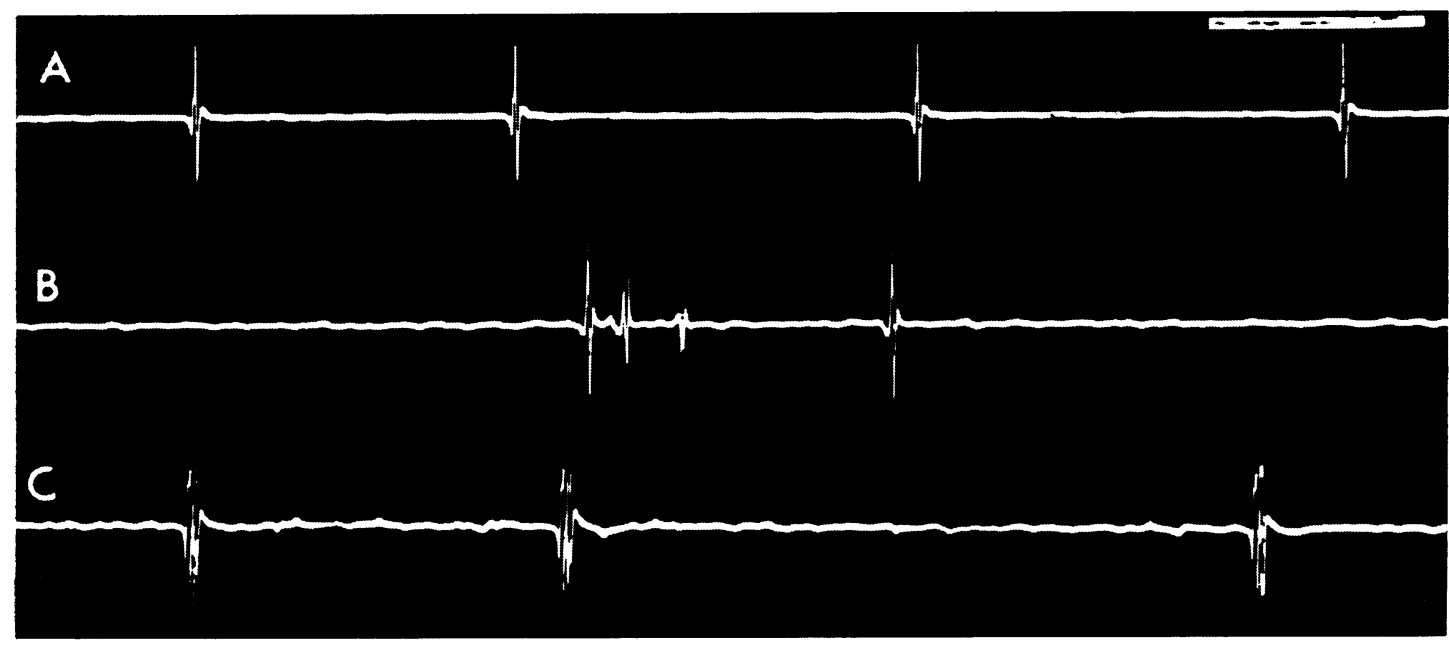

FIG. 3. Change from single discharges of one unit to bursts of several units which finally become synchronized upon fatigue. A. Before fatigue. B. Upon moderate fatigue and moderate voluntary effort. C. Upon setere fatigue and extraordinary voluntary effort. Time bar $100 \mathrm{msec}$. 


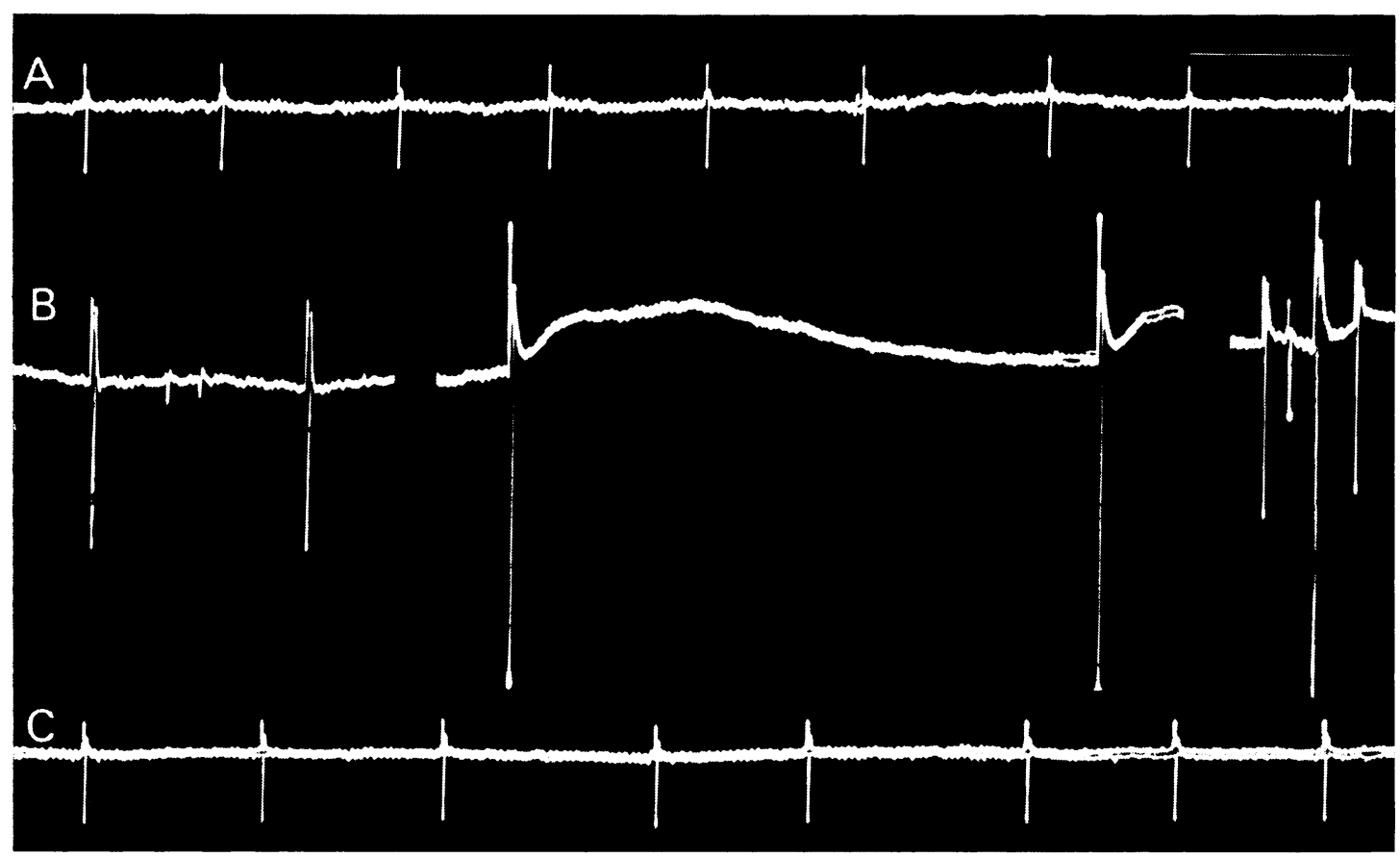

FIG. 4. Restitution of original recruitment order by reflex support of the voluntary drive. A. Before fatigue. B. Upon fatigue without reflex support. C. Upon fatigue with reflex support by a long-lasting facilitating skin stimulus in the hollow of the foot. Time bar $100 \mathrm{msec}$.

The endurance of the $\mathrm{Bl}$ unit decreases the faster it is driven. The total number of voluntary B1 discharges may decrease markedly with increasing frequency. Thus, in one experiment the subject could keep the B1 unit continuously active for 10 minutes at a frequency of $5-6 / \mathrm{sec}$, but for less than one minute if the frequency was increased to $8-9 /$ sec.

The patient's ability to activate the B1 unit voluntarily reappears after a period of rest. Several minutes' rest is required for complete restitution of endurance. The shorter the time the subject is allowed to rest, the more rapidly the Bl unit is fatigued.

On average, the endurance of the $\mathrm{B} 1$ unit is better in patients whose maximum power is 20 $25 \%$ of that of normal subjects than it is in patients whose maximum power is only $5-10 \%$ of the normal power. There are, however, subjects who diverge considerably from this general rule. Studies are in progress of the correlation between maximum power and endurance in patients with different types of lesions and different degrees of spasticity. Preliminary results indicate that fatigue appears more rapidly on bilateral than on unilateral spastic paresis.

When the patient is no longer able to keep the Bl unit in continuous activity, one of the following four phenomena may appear upon strong voluntary effort:

1. No electromyographic activity is recorded when the B1 unit no longer discharges. This also happens when fatigue is caused by driving of the B1 unit alone. The ability for voluntary activation of the B2 unit and the B3 unit is thus lost even though none of the units has been active.

2. The B1 unit is replaced by another unit. In some experiments the new unit discharges within the same frequency range as the B1 unit. In other experiments, however, the new unit discharges at a significantly higher frequency. In Fig. 1 the B1 unit (top) discharges at regular 
intervals at a frequency of $5 / \mathrm{sec}$ and is not prone to discharge at shorter intervals than $50 \mathrm{msec}$. The new unit (bottom), however, does not discharge at regular intervals until a frequency about $20 / \mathrm{sec}$ and it readily discharges at intervals of $20 \mathrm{msec}$ or less. The difference in discharge pattern is also present when the two units are temporarily active simultaneously (middle), indicating fundamental difference in motor unit type (cf. Discussion). The high-frequency unit usually discharges discontinuously without breaks when the patient makes an extra strong voluntary effort. It ceases immediately when the patient's voluntary effort is not maximal. Within 10 to 20 seconds the patient loses his ability to activate also the high-frequency unit.

3. The B1 unit is replaced by a number of units with no stable mutual recruitment order. In Fig. 2 the B1 unit continuously has a lower threshold than other units before fatigue (A), while, now one, now another and now a third unit has the lowest threshold after fatigue (B-E). The activity with unstable recruitment order appears discontinuously with long pauses. The activity periods tend to appear when the subject makes an extra strong voluntary effort. Each period of activity lasts a few seconds. Usually a patient cannot manage more than 5 to 10 such periods.

4. The B1 unit is replaced by highly synchronized bursts of several units. The synchronization phenomenon, however, appears only in patients who are able and willing to produce extraordinary voluntary effort. In Fig. 3 the B1 unit $(A)$ is upon fatigue replaced by bursts of several units (B) which upon maximum effort become highly synchronized (C). Synchronized bursts never appear for more than a few seconds.

RESTITUTION OF VOLUNTARY POWER Finally all voluntary power is lost. Rest is then the only way in which the patient can recover it. Some contraction ability is restored after only a few seconds. With such a short rest, however, the recruitment order is so indefinite that it cannot be anticipated which unit will discharge. Later on the B1 unit is regularly the unit of the lowest threshold.

If instead the subject continues voluntary effort, restitution of voluntary power is obstructed. If the $\mathrm{B} 1$ and the $\mathrm{B} 2$ units are driven together, the subject loses the B2 unit earlier than the B1 unit. If he then rests for a few seconds he is again able to activate both units voluntarily. If, however, he continues to drive the $\mathrm{B} 1$ unit alone, the B2 unit remains impossible for him to activate. Rapid reappearance upon rest but obstructed reappearance upon continuous voluntary effort should also be valid for the B1 unit. It is, however, difficult to show this, as the patient cannot continue voluntary effort without feedback from some unit in the loudspeaker.

REFLEX SUPPORT OF VOLUNTARY POWER We have considered it pertinent to study whether the fatigued B1 unit discharge can be restored by physiotherapeutic methods for reinforcement of voluntary power.

The principal method for proprioceptive neuromuscular facilitation is resistance to the contraction adjusted in such a way that the facilitating muscle-spindle system is activated more than is the inhibiting Golgi system. If the muscle is stretched when the B1 unit is replaced by units characteristic of fatigue, the B1 unit discharge tends to reappear. This favourable effect of stretch is, however, only temporary. Moreover, stretch has no effect if it is applied when the patient has lost all voluntary activity.

Primary endings of muscle spindles are very sensitive to vibration (Granit and Henatsch, 1956). Mechanical vibration of the tendon of a human muscle with a frequency of $100-400 / \mathrm{sec}$ causes a tonic reflex (De Gail et al., 1966; Hagbarth and Eklund, 1966). This reflex has been used to reinforce voluntary power in patients with spastic pareses. When the vibrator is applied to the tendon just when the patient can no longer drive his B1 unit voluntarily, the B1 unit discharge reappears.

The B1 unit discharge thus evoked is also maintained if the patient ceases his voluntary effort. When the vibrator is thereafter removed, the patient can continue the B1 unit activity voluntarily as after a period of rest. Thus, the patient's ability to activate the Bl unit voluntarily reappears on resting the voluntary drive, irrespective of whether the B1 unit is active or not. It is thus the voluntary drive and not the B1 unit that must recover.

Exteroceptive afferent activity, too, has been used for facilitation of voluntary activity in patients with spastic paresis. If the long-lasting 
nociceptive stimulus in the hollow of the foot is applied when the B1 unit discharge in the anterior tibial muscle is fatigued and replaced by other units, the B1 unit regains its dominant role. In Fig. 4 the $B 1$ unit $(A)$ is replaced by two other alternating units upon fatigue (B), but the B1 unit is again the unit of lowest threshold on tonic reflex support of the voluntary drive (C). Consequently, some patients have a significantly better endurance if they wear shoes during the experiment than if they are barefoot.

\section{DISCUSSION}

This investigation is based on the difference in the recruitment order of motor units that normally exists between tonic and phasic voluntary activity. In tonic activity the motor units are recruited in a constant order, and a unit which tends to discharge at a low frequency is always recruited before a unit which tends to discharge at a higher frequency. In phasic activity, however, the recruitment order varies with the properties of the eliciting impulse and with the preexisting state of facilitation, and a high-frequency unit can be recruited before a low frequency unit.

In this paper the fatigue reaction on voluntary driving of the anterior tibial muscle in patients with spastic paraparesis is studied. It is shown that the low-frequency unit with the lowest threshold in tonic voluntary activity can be driven only for limited periods in paretic patients, while in normal subjects it seems non-fatiguable. By driving only one unit the paretic patient can lose his ability to drive other units also. When a unit can no longer be activated voluntarily, it is still active in reflexes. We conclude that it is not the active unit itself but the voluntary drive that is fatigued.

Upon strong voluntary effort the lost lowfrequency unit can be replaced by bursts of other units. These units have no stable mutual recruitment order; they tend to be synchronized; some of them discharge at a high frequency. Unstable recruitment order, total synchronization, and predominance of high-frequency units normally appear only in phasic activity.

We consider that the most likely explanation of this change from 'tonic' to 'phasic' recruitment order is that the path for tonic voluntary facilitation of the anterior tibial muscle is inhibited or is fatigued in spastic paresis and that the patient then has to depend on a path for facilitation that normally is used only for phasic voluntary activity. The level of the presumed interference with tonic voluntary drive is, however, unknown.

The units which normally have low threshold in tonic voluntary activity discharge within a frequency range of 7-25/sec and need a long contraction time to produce maximum tension (Hannerz, 1972). The units dominating tonic voluntary activity in spastic patients before fatigue have similar discharge characteristics and are probably also of slow-twitch type.

The units which normally have low threshold only in phasic voluntary activity have higher discharge frequency ranges and probably shorter contraction times. Some of the units appearing after fatigue in spastic paraparesis also attain high discharge frequencies and may be of fast twitch type.

An alternative explanation of the high discharge frequencies of the units appearing upon fatigue is that the mechanisms regulating their discharge frequency are impaired by the pathological process. However, the units dominating before fatigue show no signs of disturbed frequency regulation even when fatigue appears. Moreover, it seems unlikely that single motor units upon voluntary driving would attain higher frequencies after a central motoneurone lesion than they could reach before the lesion. The findings thus indicate that the relative role of fast-twitch flexor motor units may increase upon fatigue in spastic patients.

Tonic proprioceptive reflex facilitation restores precisely the original recruitment order. The same normalizing effect would appear if the subject were able to increase the muscle spindle discharge by increasing his tonic voluntary innervation of gamma motoneurones.

We have shown in a previous paper that the normal recruitment order in tonic voluntary activity with low-frequency units coming before units of higher frequency can be disturbed by blocking the gamma loop with lidocaine injections in the muscle nerve or local cooling of the muscle (Grimby and Hannerz, 1973a). Freyschuss and Knutsson (1971) have shown that blockades of the extra- and intrafusal muscle fibres with celocurine or curare in normal man result in 
inability to maintain voluntary discharge in at least large alpha motoneurone fibres. There are thus indications that gamma loop insufficiency may be of significance for the fatigue reaction in subjects with central motoneurone lesions. It is not, however, possible to decide what role insufficiency of the voluntary gamma motoneurone innervation has in comparison with insufficiency of the direct alpha motoneurone innervation.

Increased fatiguability of voluntary activity may appear in various situations where voluntary drive is insufficient. A change from 'tonic' to 'phasic' recruitment order upon fatigue indicating a selective insufficiency of tonic voluntary drive has not, however, been seen normally or in other diseases than spastic paresis. Parkinsonism patients, for instance, never change from 'tonic' to 'phasic' recruitment order without an intervening period of rest (Grimby and Hannerz, 1973b).

A corresponding single unit study of longlasting voluntary activity of the soleus muscle is in progress. Preliminary results indicate that the endurance of low-frequency units is better in the soleus than in the anterior tibial muscle. This agrees with findings in spastic paresis that type I muscle fibres, which presumably belong to lowfrequency units, are atrophic as after disuse in the weakly spastic flexor muscles, but escape atrophy in the strongly spastic extensor muscles in the leg (Edström et al., 1973). Further experiments are needed before it can be decided what role spasticity and inhibition from extensor muscles play in the fatigue in the anterior tibial muscle.

Our results should be also of practical physiotherapeutic interest. The normalizing effect on the recruitment order obtained by tonic reflex support of the voluntary drive gives a rational basis for those types of physiotherapy in which tonic reflexes are used to reinforce voluntary effort. The original contraction ability lost upon fatigue rapidly reappears upon rest. If instead voluntary effort is continued, this reappearance is obstructed. This stresses the importance of optimal alternation between activity and rest. The same restitution of the original voluntary contraction ability that is obtained upon rest can be obtained if the voluntary activation is temporarily replaced by reflex activation. It is thus urgent to develop practical methods for intermittent reflex facilitation that can disburden the voluntary drive in patients with central motoneurone lesions. Mårtensson et al. (1969) have described a method having this effect. They have succeeded in bringing about a considerable improvement of the anterior tibial muscle function in walking by using in the hollow of the foot a pelot that in each stance phase causes a flexion reflex of appropriate duration. As, the favourable effect of reflex support seems to be obtained through a saving of the voluntary drive, there should be no need for precise adjustment of reflex strength and duration. This should make it easier to develop practical methods.

\section{REFERENCES}

De Gail, P., Lance, J. W., and Neilson, P. D. (1966). Differen tial effects on tonic and phasic reflex mechanisms produced by vibration of muscles in man. Journal of Neurology. Neurosurgery, and Psychiatry, 29, 1-11.

Edström, L., Grimby, L., and Hannerz, J. (1973). Correlation between recruitment order of motor units and muscle atrophy pattern in central motoneurone lesion: significancer of spasticity. Experientia, 29, 560-561.

Freyschuss, U., and Knutsson, E. (1971). Discharge patternse in motor nerve fibres during voluntary effort in man.? Acta Physiologica Scandinavica, 83, 278-279.

Granit, R., and Henatsch, H. D. (1956). Gamma control of dynamic properties of muscle spindles. Journal of Neurophysiology, 19, 356-366.

Grimby, L., and Hannerz, J. (1973a). Tonic and phasic recruitment order of motor units in man under normal and pathological conditions. In New Developments in Electromyography and Clinical Neurophysiology, vol. 3, pp. 225233. Edited by J. E. Desmedt. Karger: Basel.

Grimby, L., and Hannerz, J. (1973b). Disturbances of the recruitment order of anterior tibial motor units in bradykinesia of Parkinsonism. Journal of Neurology, Neurosurgery, and Psychiatry. (In press.)

Hagbarth, K.-E., and Eklund, G. (1966). Motor effects of vibratory muscle stimuli in man. In Muscular Afferents and Motor Control. Proceedings of the First Nobel Symposium, 1965, Södergarn. Edited by R. Granit. Almqvist and Wiksell: Stockholm.

Hannerz, J. (1973). Discharge properties of motor units in man. Experientia, 29, 45-46.

Mårtensson, A., Knutsson, E., and Hindmarsh, J. (1969). Gait facilitation by plantar punctate pressure stimulation in spastic paraparesis. Scandinavian Journal of Rehabilitation and Medicine, 1, 147-148.

Merton, P. A. (1954). Voluntary strength and fatigue. Journal of Physiology, 123, 553-564. 